Submitted to Phys Rev E, July 41996

\title{
Electroconvection in a Suspended Fluid Film: A Linear Stability Analysis
}

\author{
Zahir A. Daya and Stephen W. Morris \\ Department of Physics and Erindale College \\ University of Toronto \\ 60 St. George St., \\ Toronto, Ontario, Canada M5S 1A7 \\ John R. de Bruyn \\ Department of Physics \\ Memorial University of Newfoundland \\ St. John's, Newfoundland, Canada A1B $3 X^{r}$
}

(November 10, 2018)

\begin{abstract}
A suspended fluid film with two free surfaces convects when a sufficiently large voltage is applied across it. We present a linear stability analysis for this system. The forces driving convection are due to the interaction of the applied electric field with space charge which develops near the free surfaces. Our analysis is similar to that for the two-dimensional Bénard problem, but with important differences due to coupling between the charge distribution and the field. We find the neutral stability boundary of a dimensionless control parameter $\mathcal{R}$ as a function of the dimensionless wave number $\kappa$. $\mathcal{R}$,
\end{abstract}


which is proportional to the square of the applied voltage, is analogous to the Rayleigh number. The critical values $\mathcal{R}_{c}$ and $\kappa_{c}$ are found from the minimum of the stability boundary, and its curvature at the minimum gives the correlation length $\xi_{0}$. The characteristic time scale $\tau_{0}$, which depends on a second dimensionless parameter $\mathcal{P}$, analogous to the Prandtl number, is determined from the linear growth rate near onset. $\xi_{0}$ and $\tau_{0}$ are coefficients in the Ginzburg-Landau amplitude equation which describes the flow pattern near onset in this system. We compare our results to recent experiments.

Typeset using REVTEX 


\section{INTRODUCTION}

The regular patterns which form when a dissipative, nonequilibrium system is driven just beyond the threshold of certain symmetry-breaking instabilities are in many ways analogous to the simple ordered phases which appear following equilibrium phase transitions [1]. Patterns can, however, exhibit interesting nonlinear dynamical behavior, for example chaotic motion, which has no analog in equilibrium systems. Several fluid-dynamical systems undergo pattern-forming instabilities which are amenable to both theoretical and laboratory study. Examples which have been extensively explored into the nonlinear regime include Rayleigh-Bénard convection [1,2], Taylor vortex flow [1,22], and electroconvection in nematic liquid crystals [3]. In each of these cases, an essential foundation for understanding the nonlinear behavior is a complete analysis of the initial linear instability. The linear stability analyses for Rayleigh-Bénard convection and Taylor vortex flow are classic problems in fluid mechanics [1, [4, 5]. The mechanism of the linear instability for electroconvection in nematic liquid crystals required many years to elucidate, but even this very complex system is now reasonably well understood in both the linear and the nonlinear regimes [3].

Our objective in this paper is to carry out a realistic linear stability analysis for a different electrically driven instability, namely electroconvection in a thin, suspended fluid film. We have observed electroconvection patterns in experiments on thin suspended films of smectic A liquid crystals [6, [, 8, 9, 10], which are isotropic in the plane of the film but have a layered structure which very strongly impedes flows perpendicular to the film. As a result, these films can convect rapidly with no change in thickness. We have observed convection in films only a few molecules thick. Our immediate goal is to understand the onset of electroconvection in this system. The instability mechanism we describe is, however, not specific to smectic films and would presumably apply to any sufficiently two-dimensional, weakly conducting fluid film. In fact, similar convective flows have been observed in thicker films of nematic and isotropic liquids [11]. In these cases, however, surface tension effects and the convective flow itself cause thickness variations in the films, which make their behavior more complicated 
than that of the smectic films.

The model we describe below is physically similar to a highly simplified one proposed by Faetti, Fronzoni and Rolla [11] for the "vortex mode" convection they observed in nematic films, but our analysis is carried much farther. There is also some similarity between the driving mechanism considered here and that which drives electroconvection near the free surface in a partly-filled capacitor [12].

The relevant experimental arrangement is shown schematically in Fig. 11. A thin fluid film is suspended between electrodes, with both its top and bottom surfaces free. The width of the film $d$ is much larger than its thickness $s$, and we will treat it as being purely twodimensional. When the de voltage applied across the electrodes exceeds a critical value, the film convects in a pattern of vortices confined to the plane of the film. We neglect any effects of air drag by assuming that the film is suspended in vacuum. We will also treat the film as a weak ohmic conductor, and neglect any electrochemical charge production on the electrodes or in the bulk of the fluid. The currents involved are assumed to be sufficiently small that magnetic forces are insignificant.

The body force responsible for driving any electroconvective flow results from an electric field acting on regions of nonzero charge density in the fluid. To analyze the electroconvection system we must first identify the mechanism which gives rise to regions of locally unbalanced charge in the fluid, and second, solve for the charges and fields selfconsistently, since these are coupled by Maxwell's equations. In our model, the charge density arises due to the electrical boundary conditions at the free surfaces of the film [8]. The inset to Fig. 11 shows the essential details of the charge separation mechanism. Below the onset of convection, the applied voltage drives a uniform, steady current density $\overrightarrow{\mathbf{J}}$ through the film. This is accompanied by a constant electric field $\overrightarrow{\mathbf{E}}_{\text {inside }}=\overrightarrow{\mathbf{J}} / \sigma$, where $\sigma$ is the bulk conductivity. The interior field $\overrightarrow{\mathbf{E}}_{\text {inside }}$ has no component perpendicular to the film plane. However, the exterior field $\overrightarrow{\mathbf{E}}_{\text {outside }}$ must have both parallel and perpendicular components just outside the free surfaces of the film. It cannot in general be perpendicular to the surface because the surface is not an equipotential: the film is an ohmic conductor, so its surface potential 
varies linearly with the coordinate between the electrodes. The parallel component $\overrightarrow{\mathbf{E}}_{\text {outside }}^{\|}$ is equal to $\overrightarrow{\mathbf{E}}_{\text {inside }}$ by the usual matching conditions on electric fields across surfaces. The perpendicular component $\overrightarrow{\mathbf{E}}_{\text {Outside }}^{\perp}$ is proportional to the surface charge density $\alpha$ at that location. It is the interaction of the parallel component of the field $\overrightarrow{\mathbf{E}}_{\text {inside }} \equiv \overrightarrow{\mathbf{E}}_{\text {outside }}^{\|}$with the surface charge density $\alpha$ at the two free surfaces that drives the convective flow above the onset of the instability.

In Section III, we calculate the surface charge density below onset by solving for the fields exterior to the film. This problem is solved analytically for thin films in two simple electrode geometries. We find that a "charge inversion" is set up in the base state: the film has a positive charge density close to the positive electrode, and a negative charge density close to the negative electrode. This inverted charge distribution is sustained by the applied potential difference across the conductor — without a potential difference, the film surfaces are equipotentials, the component of the field parallel to the surface is zero, and thus there are no forces to drive convection, even if an electrostatic surface charge is present. This inverted base state configuration is analogous to the mass density inversion that arises in the Bénard problem, and in Section [II] we show that it leads to a hydrodynamic instablity when the applied voltage is sufficiently large. Unlike the density in the Bénard problem, however, the charge density in the base state is a nonlinear function of position across the film.

By treating the film as two-dimensional, we neglect the diffusion of charge on the scale of the film thickness $s$, which acts to smear the surface charge over a thickness of order the Debye screening length $\lambda_{D}[\mathbb{8}]$. For the very thin films considered here, $\lambda_{D}$ may be comparable to $s$, in which case the surface charges and surface forces described above will extend over the whole thickness of the film. One can show that the total charge contained in one such Debye layer is the same as that which would reside at one surface in the absence of diffusion. The approximation that the film is a two-dimensional conducting sheet may be expected to break down for thick films for which $s \gg \lambda_{D}$. In this limit, surface forces may 
lead to significant shears and internal flows, as is apparently the case in thick nematic and isotropic films [11]. Diffusion on the much larger scale of the film width $d$ is also neglected.

In Section III, we describe the linear stability analysis for infinitesimal perturbations about the base state. The stability calculation is somewhat analogous to that for the Bénard problem. Dimensionless quantities $\mathcal{R}$ and $\mathcal{P}$ appear, which are analogous to the Rayleigh and Prandtl numbers. $\mathcal{R}$ is proportional to the square of the applied voltage, while $\mathcal{P}$ is the ratio of the thin film charge relaxation time to the viscous relaxation time.

The differences between our calculation and the Bénard problem are due to the additional coupling between the field and the charge density, which is also responsible for the nonlinearity of the base state charge density. The charge density and the field are analogous to the mass density and the gravitational acceleration in the Bénard problem; the new requirement that these also satisfy Maxwell's equations amounts to requiring a nonlocal relation between the charge density and the electric potential. If we suppress this nonlocality by assuming these are simply proportional, the base state charge density becomes linear and our problem reduces completely to the Bénard case. Interestingly, this proportionality is nearly correct except near the edges of the film.

In Section $1 \nabla$, we discuss the results for the neutral curve, and compare the predictions for the critical voltage $V_{c}$ and the critical wave number $\kappa_{c}$ to the values obtained from experiments. We also calculate the correlation length $\xi_{0}$ from the curvature of the neutral curve near $\kappa_{c}$ and the characteristic time $\tau_{0}$ from the linear growth rate at $\kappa_{c}$. These quantities, which are coefficients in the Ginzburg-Landau equation which describes the amplitude of the pattern near onset, as discussed below [10,13], are also compared to experimental results. Section $\nabla$ is a brief summary and conclusion.

\section{THE BASE STATE CHARGE DENSITY}

Our first task is to calculate the configuration of charges and fields below the onset of convection. As described in the previous section, this is essentially an electrostatic problem 
in the region exterior to the film. The coordinates used and the geometry of the electrodes and film are shown in Fig. 2. The origin is at the centre of the film, which lies between $z= \pm s / 2$ and $y= \pm d / 2$. We will consider the limit of a thin film for which $s \ll d$. The film is assumed to extend infinitely in the $x$ direction. The upper and lower surfaces of the film are free and the region outside the film has permittivity $\epsilon_{0}$. The permittivity $\epsilon$ of the fluid will turn out to be irrelevant to the analysis of the base state.

The film will be treated as a charged conducting sheet of negligible thickness in the $x y$ plane with bulk conductivity $\sigma$. Its edges at $y= \pm d / 2$ are held at applied potentials $\pm V / 2$ by electrodes of zero thickness. Below the onset of convection, the film behaves as an ohmic conductor, so that the potential on the film varies linearly between $-V / 2$ and $+V / 2$ for $-d / 2 \leq y \leq d / 2$. The potential is zero on the $x$ axis and as $|z| \rightarrow \infty$. The potential exterior to the film is symmetric above and below the $x y$ plane and independent of $x$. The charge density $q$ is proportional to the perpendicular component of the field exterior to the film, at the film's surface, and so to the $z$ derivative of the potential there. To calculate $q$ we need only solve for the potential in the upper half of the $y z$ plane, subject to boundary conditions on the $y$ axis. We will consider two simple electrode geometries, which we refer to as "plates" and "wires". In the plate geometry, we specify the potential on the rest of the $y$ axis to be $-V / 2$ for $y<-d / 2$ and $+V / 2$ for $y>d / 2$. Solving for the potentials is a Dirichlet problem which we solve below using a Green function. This geometry corresponds to a film held between infinite knife edges. Most of the experiments [6,8.9.10], however, used thin wire electrodes to support the edges of the film, as shown schematically in Fig. [1(a). To model this geometry, the applied potential $\pm V / 2$ is specified only at the two points $y= \pm d / 2$. For $|y|>d / 2$ we require that the $z$ derivative of the potential on the $y$ axis be zero. Thus, in the upper half of the $y z$ plane, we must solve a mixed boundary value problem with Dirichlet conditions for $-d / 2 \leq y \leq d / 2$ and Neumann conditions for $|y|>d / 2$. This is done analytically below, using the method of dual integral equations [14]. 


\section{A. The Base State for Plate Electrodes}

We begin with the simpler plate electrode configuration. We must solve the Laplace equation for the potential $\Psi$ in the upper half $y z$ plane,

$$
\left(\frac{\partial^{2}}{\partial y^{2}}+\frac{\partial^{2}}{\partial z^{2}}\right) \Psi(y, z)=0
$$

subject to the piecewise linear Dirichlet boundary conditions on the $y$ axis

$$
\begin{aligned}
\Psi(y, 0) & =-\frac{V}{2} & & -\infty<y \leq-\frac{d}{2} \\
& =\frac{V}{d} y & & -\frac{d}{2}<y<\frac{d}{2} \\
& =\frac{V}{2} & & \frac{d}{2} \leq y<\infty .
\end{aligned}
$$

The appropriate Green function is constructed from a unit line charge at $\left(y^{\prime}, z^{\prime}\right)$ and its image at $\left(y^{\prime},-z^{\prime}\right)$

$$
G\left(y, z ; y^{\prime}, z^{\prime}\right)=-\log \frac{\left(y-y^{\prime}\right)^{2}+\left(z-z^{\prime}\right)^{2}}{\left(y-y^{\prime}\right)^{2}+\left(z+z^{\prime}\right)^{2}}
$$

The potential at any point in the upper half plane is given by

$$
\Psi(y, z)=\left.\frac{1}{4 \pi} \int_{-\infty}^{\infty} \Psi\left(y^{\prime}, 0\right) \frac{\partial G}{\partial z^{\prime}}\right|_{z^{\prime}=0} d y^{\prime}=\frac{z}{\pi} \int_{-\infty}^{\infty} \frac{\Psi\left(y^{\prime}, 0\right)}{z^{2}+\left(y-y^{\prime}\right)^{2}} d y^{\prime}
$$

The surface charge density on the film is a consequence the fact that the $z$ components of the electric fields inside and outside the conducting film are different. Inside the conductor, in the absence of diffusion, the $z$ component of the field is identically zero, as in Fig. 1(b), and hence only the external field is required to determine the surface charge density. As mentioned in the Introduction, the presence of a diffusion layer near the surface does not change the total charge density present, per unit area. The surface charge density on upper side of the film is $-\epsilon_{0} \partial \Psi /\left.\partial z\right|_{z=s / 2}$. In the limit $s \rightarrow 0, \partial \Psi / \partial z$ is discontinuous across $z=0$, so we use a one-sided derivative valid as $z \rightarrow 0^{+}$. To get the total surface charge density $q$ on the film, we introduce a factor of two to account for the two free surfaces, so that

$$
q=-\left.2 \epsilon_{0} \frac{\partial \Psi}{\partial z}\right|_{z=0^{+}}
$$


Using this with Eq. (2.4), interchanging the order of differentiation and integration, and using Eq. (2.2) gives

$$
\begin{gathered}
-\frac{\pi}{2 \epsilon_{0}} q_{p}(y)=\left.\int_{-\infty}^{-\frac{d}{2}} \frac{-V / 2}{z^{2}+\left(y-y^{\prime}\right)^{2}} d y^{\prime}\right|_{z=0^{+}}+\left.\int_{-\frac{d}{2}}^{\frac{d}{2}} \frac{V y^{\prime} / d}{z^{2}+\left(y-y^{\prime}\right)^{2}} d y^{\prime}\right|_{z=0^{+}} \\
+\left.\int_{\frac{d}{2}}^{\infty} \frac{V / 2}{z^{2}+\left(y-y^{\prime}\right)^{2}} d y^{\prime}\right|_{z=0^{+}}
\end{gathered}
$$

where the subscript $p$ denotes plate electrodes. After integration, the resulting expression was expanded as a power series in $z$ and evaluated as $z \rightarrow 0^{+}$. After some simplification, we find the surface charge density for the case of plate electrodes to be given by

$$
q_{p}(y)=-\frac{2 V \epsilon_{0}}{\pi d} \log \left|\frac{y-d / 2}{y+d / 2}\right|
$$

This distribution is shown in Fig. 3. Note that the charge density is positive near the positive electrode and negative near the negative electrode, giving the charge inversion described in the Introduction. The charge density diverges at the electrodes, which is an unphysical consequence of the limit $s \rightarrow 0$. In the real system, the finite thicknesses of the film and electrodes will impose a cutoff on $q_{p}$. This divergence, while unphysical, is weak enough to be mathematically tractable. It will turn out that the rigid boundary conditions we impose on the flow, described in the next section, ensure that the contributions from the edges of the film are small. On the other hand, the fact that $q_{p}(y)$ is not a linear function of $y$ has important consequences for the quantitative results of the stability analysis.

\section{B. The Base State for Wire Electrodes}

We now turn to the case of wire electrodes. For this case, the mixed boundary value electrostatic problem can be solved by the theory of dual integral equations [14]. We must solve Eq. (2.1), subject to the mixed boundary conditions

$$
\begin{aligned}
\Psi(y, 0) & =\frac{V}{d} y & \text { for } & & |y| \leq d / 2 \\
\left.\frac{\partial \Psi(y, z)}{\partial z}\right|_{z=0^{+}} & =0 & \text { for } & & |y|>d / 2 .
\end{aligned}
$$


By separation of variables and using the fact that $\Psi(0,0)=0$, we make the ansatz that the potential in the upper half plane can be written as

$$
\Psi(y, z)=\int_{0}^{\infty} \frac{A(k)}{k} e^{-k z} \sin (k y) d k .
$$

With this ansatz, we find the dual integral equations

$$
\begin{array}{rlrl}
\int_{0}^{\infty} \frac{A(k)}{k} \sin (k y) d k & =\frac{V}{d} y \quad \text { for } & & |y|<d / 2 \\
\int_{0}^{\infty} A(k) \sin (k y) d k & =0 \quad \text { for } \quad & |y|>d / 2 .
\end{array}
$$

This pair of integral equations may be solved for the potential in the upper half plane by the method of Sneddon [14], giving

$$
\Psi(y, z)=\frac{V}{2} \int_{0}^{\infty} \frac{J_{1}(k d / 2)}{k} e^{-k z} \sin (k y) d k,
$$

where $J_{1}$ is the first order Bessel function of the first kind. Using Eq. (2.5), the total surface charge density is given by

$$
q_{w}(y)=\epsilon_{0} V \int_{0}^{\infty} J_{1}(k d / 2) \sin (k y) d k=\frac{2 \epsilon_{0} V}{d} \frac{y}{\sqrt{(d / 2)^{2}-y^{2}}} .
$$

The subscript $w$ denotes wire electrodes. This result is also shown in Fig. 3. As for the plate electrodes, we find an inverted charge distribution and divergences at the edges of the film.

Below the onset of convection, the electric field inside the film is constant and points along $-\hat{\mathbf{y}}$. The force driving the convection is due to the in-plane electric field acting on the charge densities $q$. Unlike the Bénard problem, in which the temperature profile is linear below onset, here the charge density, and hence the body force, is not linear in $y$. This has the effect of introducing certain non-constant coefficients into the stability problem, as described in the next section.

\section{LINEAR STABILITY ANALYSIS}

In this Section we consider the stability of the base state to infinitesimal perturbations. We will show that for sufficiently large $V$, the electrical forces overcome viscous and con- 
duction losses and the film becomes unstable to convection.

\section{A. The Perturbation Equations}

Within the thin film, we assume that the fluid velocity $\overrightarrow{\mathbf{u}}$ is confined to the film plane, with $\overrightarrow{\mathbf{u}}=u \hat{\mathbf{x}}+v \hat{\mathbf{y}}$. In addition, we will neglect any shears in the $z$ direction. As discussed above, these assumptions are reasonable in the context of thin smectic films, where the layer structure strongly inhibits flow across layers. We treat the film as a two-dimensional conducting fluid, with areal material parameters $\rho_{s}=s \rho, \eta_{s}=s \eta, \sigma_{s}=s \sigma$, where $s$ is the film thickness and $\rho$ is the bulk density, $\eta$ is the bulk molecular viscosity, and $\sigma$ is the bulk

conductivity. (In smectic A films, the viscosity is highly anisotropic; the relevant component to use for $\eta$ is $\eta_{3}$, the viscosity related to shears within layer planes.) The two-dimensional pressure field is given by $P_{s}=s P$. The flow is driven by the surface force density $q \overrightarrow{\mathbf{E}}_{s}$, where $\overrightarrow{\mathbf{E}}_{s}$ is the electric field in the film plane. The flow velocity is governed by the two-dimensional Navier-Stokes equation

$$
\rho_{s}\left[\frac{\partial \overrightarrow{\mathbf{u}}}{\partial t}+\left(\overrightarrow{\mathbf{u}} \cdot \nabla_{s}\right) \overrightarrow{\mathbf{u}}\right]=-\nabla_{s} P_{s}+\eta_{s} \nabla_{s}^{2} \overrightarrow{\mathbf{u}}+q \overrightarrow{\mathbf{E}}_{s}
$$

where $\nabla_{s}$ is the two-dimensional gradient $\hat{\mathbf{x}} \partial / \partial x+\hat{\mathbf{y}} \partial / \partial y$. The fluid is assumed to be incompressible, so that

$$
\nabla_{s} \cdot \overrightarrow{\mathbf{u}}=0
$$

This condition may also be viewed as a constant thickness assumption. We impose physically realistic rigid boundary conditions on $\overrightarrow{\mathbf{u}}$ at the edges of the film, so

$$
\overrightarrow{\mathbf{u}} \equiv 0 \quad \text { and } \quad \frac{\partial v}{\partial y} \equiv 0 \quad \text { at } \quad y= \pm d / 2
$$

The motion of charge is governed by the charge continuity equation

$$
\frac{\partial q}{\partial t}=-\nabla_{s} \cdot \overrightarrow{\mathbf{J}}_{s}=-\nabla_{s} \cdot\left(q \overrightarrow{\mathbf{u}}+\sigma_{s} \overrightarrow{\mathbf{E}}_{s}\right)
$$


in which $\overrightarrow{\mathbf{J}}_{s}$ is the two-dimensional current density in the plane of the film, and includes contributions from both conduction $\left(\sigma_{s} \overrightarrow{\mathbf{E}}_{s}\right)$ and convection $(q \overrightarrow{\mathbf{u}})$. Diffusion of charge in the plane of the film has been neglected.

The electric field in the plane of the film $\overrightarrow{\mathbf{E}}_{s}$ is given by

$$
\overrightarrow{\mathbf{E}}_{s}=-\nabla_{s} \Psi_{s}=-\left.\nabla_{s} \Psi\right|_{z=0} .
$$

As in the previous section, $\Psi$ is the potential which solves the three-dimensional Laplace equation,

$$
\nabla^{2} \Psi=0
$$

in the half space $z \geq 0$, with the surface charge density given by Eq. (2.5).

The surface charge density $q$ in Eqs. (3.1) and (3.4) in principle contains both the density of free charges $q_{\text {free }}$ and that of the dielectric polarization charges, so that $q=q_{\text {free }}-\nabla_{s} \cdot \overrightarrow{\mathbf{P}}_{s}$. Here $\overrightarrow{\mathbf{P}}_{s}=s \overrightarrow{\mathbf{P}}$, where $\overrightarrow{\mathbf{P}}$, the bulk polarization density, is given by $\overrightarrow{\mathbf{P}}=\epsilon_{0} \chi \overrightarrow{\mathbf{E}}$, where $\chi$ is the electric susceptibility. Inside the film, $\overrightarrow{\mathbf{E}}=\overrightarrow{\mathbf{E}}_{s}$, independent of $z$, and has zero $z$ component. Eq. (2.5), which only involves exterior fields, therefore only gives $q_{\text {free }}$. In the base state, however, $\nabla_{s} \cdot \overrightarrow{\mathbf{E}}_{s}=0$ and $q=q_{\text {free }}$, so polarization effects are irrelevant. In the general case, one can show using the scalings given below that the dimensionless form of the $\nabla_{s} \cdot \overrightarrow{\mathbf{P}}_{s}$ terms are proportional to $\chi s / d$. For the experiments of interest, $\chi s / d \approx 10^{-4}$, so we can safely neglect polarization effects.

Eqs. (3.1)-(3.6) have a simple solution when $\overrightarrow{\mathbf{u}} \equiv 0$. Eq. (3.6), subject to the appropriate boundary conditions, corresponds to the base state charge density problem solved in the previous section. Once the fields and $q$ are found from Eqs. (2.5) and (3.6), Eq. (3.1) can be solved for the pressure $P_{s}$ whose gradient balances the surface force density $q \overrightarrow{\mathbf{E}}_{s}$. Eq. (3.4) then gives a constant current density $\overrightarrow{\mathbf{J}}_{s}=\sigma_{s} \overrightarrow{\mathbf{E}}_{s}$, with a constant interior field $\overrightarrow{\mathbf{E}}_{s}=-(V / d) \hat{\mathbf{y}}$. This is a selfconsistent solution which corresponds to hydrostatic equilibrium. The pressure gradient points everywhere toward the midline of the film, $y=0$.

Expanding the divergence in Eq. (3.4) and using Eq. (3.2), the charge continuity equation becomes 


$$
\frac{\partial q}{\partial t}=-\overrightarrow{\mathbf{u}} \cdot \nabla_{s} q-\sigma_{s} \nabla_{s} \cdot \overrightarrow{\mathbf{E}}_{s}
$$

Note that $\nabla_{s} \cdot \overrightarrow{\mathbf{E}}_{s}$ is not equivalent to $\nabla \cdot \overrightarrow{\mathbf{E}}$, because of discontinuities in the $z$ component of $\overrightarrow{\mathbf{E}}$ at $z=0$. Thus, $\nabla_{s} \cdot \overrightarrow{\mathbf{E}}_{s}$ is not directly related to the charge density $q . \nabla_{s} \cdot \overrightarrow{\mathbf{E}}_{s}$ can be found only after solving the full three-dimensional Laplace problem given by Eq. (3.6).

To examine the stability of the base state, we introduce perturbed quantities

$$
\begin{gathered}
\overrightarrow{\mathbf{u}}=0+\overrightarrow{\mathbf{u}}^{(1)}, \\
q=q^{(0)}+q^{(1)}, \\
P_{s}=P_{s}^{(0)}+P_{s}^{(1)}, \\
\overrightarrow{\mathbf{E}}_{s}=\overrightarrow{\mathbf{E}}_{s}^{(0)}+\overrightarrow{\mathbf{E}}_{s}^{(1)} .
\end{gathered}
$$

where $\overrightarrow{\mathbf{E}}_{s}^{(0)}=E_{y}{ }^{(0)} \hat{\mathbf{y}}$ and $\overrightarrow{\mathbf{E}}_{s}^{(1)}=E_{x}{ }^{(1)} \hat{\mathbf{x}}+E_{y}{ }^{(1)} \hat{\mathbf{y}}$. Here $E_{y}{ }^{(0)}=-V / d$, and $q^{(0)}$ is the base state charge density found in the previous section. To first order in the small perturbations, Eqs. (3.1), (3.2), and (3.7) become

$$
\begin{aligned}
\nabla_{s} \cdot \overrightarrow{\mathbf{u}}^{(1)} & =0 \\
\rho_{s} \frac{\partial \overrightarrow{\mathbf{u}}^{(1)}}{\partial t} & =-\nabla_{s} P_{s}^{(1)}+\eta_{s} \nabla_{s}{ }^{2} \overrightarrow{\mathbf{u}}^{(1)}+q^{(0)} E_{x}{ }^{(1)} \hat{\mathbf{x}}+q^{(1)} E_{y}{ }^{(0)} \hat{\mathbf{y}}+q^{(0)} E_{y}{ }^{(1)} \hat{\mathbf{y}} \\
\frac{\partial q^{(1)}}{\partial t} & =-\overrightarrow{\mathbf{u}}^{(1)} \cdot \nabla_{s} q-\sigma_{s}\left[\nabla_{s} \cdot\left(E_{x}{ }^{(1)} \hat{\mathbf{x}}+E_{y}{ }^{(1)} \hat{\mathbf{y}}\right)\right] .
\end{aligned}
$$

Taking the curl of Eq. (3.13) eliminates the pressure. Taking a second curl and using Eq. (3.2) gives

$$
\rho_{s} \frac{\partial}{\partial t} \nabla_{s}^{2} \overrightarrow{\mathbf{u}}^{(1)}=\eta_{s} \nabla_{s}^{2} \nabla_{s}^{2} \overrightarrow{\mathbf{u}}^{(1)}-\nabla_{s} \times\left[\nabla_{s} \times\left(q^{(0)} E_{x}^{(1)} \hat{\mathbf{x}}+q^{(1)} E_{y}^{(0)} \hat{\mathbf{y}}+q^{(0)} E_{y}^{(1)} \hat{\mathbf{y}}\right)\right] .
$$

From this equation, we select the $y$ component, which is

$$
\rho_{s} \frac{\partial}{\partial t} \nabla_{s}^{2} v^{(1)}=\eta_{s} \nabla_{s}^{2} \nabla_{s}^{2} v^{(1)}+E_{y}^{(0)} \frac{\partial^{2} q^{(1)}}{\partial x^{2}}-\frac{\partial q^{(0)}}{\partial y} \frac{\partial E_{x}^{(1)}}{\partial x} .
$$

Using the fact that $q^{(0)}$ is only a function of $y$, Eq. (3.14) becomes

$$
\frac{\partial q^{(1)}}{\partial t}=-v^{(1)} \frac{\partial q^{(0)}}{\partial y}-\sigma_{s}\left(\frac{\partial E_{x}^{(1)}}{\partial x}+\frac{\partial E_{y}^{(1)}}{\partial y}\right) .
$$


In the previous equations, $\overrightarrow{\mathbf{E}}_{s}^{(1)}$ is the in-plane electric field produced by the charge distribution $q^{(1)}$. Introducing perturbations for the electric potential with $\Psi=\Psi^{(0)}+\Psi^{(1)}$, we have $\overrightarrow{\mathbf{E}}_{s}^{(1)}=-\nabla_{s} \Psi_{s}^{(1)}=-\left.\nabla_{s} \Psi^{(1)}\right|_{z=0}$. The perturbation potential $\Psi^{(1)}$ is the solution of a new three-dimensional Laplace problem in $z \geq 0$ analogous to Eq. (3.6),

$$
\nabla^{2} \Psi^{(1)}=0
$$

with $q^{(1)}=-\left.2 \epsilon_{0}\left(\partial \Psi^{(1)} / \partial z\right)\right|_{z=0^{+}}$.

We now replace the various field components with the appropriate derivatives of the potential in Eqs. (3.16) and (3.17), to get

$$
\begin{aligned}
\rho_{s} \frac{\partial}{\partial t} \nabla_{s}^{2} v^{(1)} & =\eta_{s} \nabla_{s}^{2} \nabla_{s}^{2} v^{(1)}+E_{y}^{(0)} \frac{\partial^{2} q^{(1)}}{\partial x^{2}}+\frac{\partial q^{(0)}}{\partial y} \frac{\partial^{2} \Psi_{s}^{(1)}}{\partial x^{2}} \\
\frac{\partial q^{(1)}}{\partial t} & =-v^{(1)} \frac{\partial q^{(0)}}{\partial y}+\sigma_{s}\left(\frac{\partial^{2} \Psi_{s}^{(1)}}{\partial x^{2}}+\frac{\partial^{2} \Psi_{s}^{(1)}}{\partial y^{2}}\right) .
\end{aligned}
$$

Eqs. (3.18)-(3.20) are the equations for the perturbations that we must solve to determine the stability of the base state.

The specification of the boundary conditions necessary to solve Eq. (3.18) requires some explanation. By writing $\Psi=\Psi^{(0)}+\Psi^{(1)}$, we split the full Laplace problem of Eq. (3.6) into separate Laplace problems at each order. At zeroth order, the boundary conditions at $z=0$ on $\Psi^{(0)}$ are those described in Section $\mathbb{1 1}$ for each electrode configuration in the base state. In particular, $\Psi^{(0)}$ was set equal to $\pm V / 2$ at the edges of the film. At first order, the boundary conditions on $\Psi^{(1)}$ require that $\Psi^{(1)}=0$ at the edges of the film, and on both of the electrodes in the plate case. In the wire electrode case we require $\partial \Psi^{(1)} /\left.\partial z\right|_{z=0^{+}}=0$ for $|y|>d / 2$ and $z=0$. In either electrode case, we will find Dirichlet boundary conditions for $\Psi^{(1)}$ on the film itself by selfconsistently solving Eq. (3.20). Proceeding in this way, the boundary conditions on the total potential $\Psi$ are satisified by the superposition of $\Psi^{(0)}$ and $\Psi^{(1)}$. 


\section{B. The Normal Mode Expansion}

We now expand the velocity, charge density and potential perturbations in normal modes which are periodic in $x$ with wavenumber $k$, and have growth rate $\gamma$,

$$
\begin{aligned}
v^{(1)} & =\Lambda(y) e^{i k x+\gamma t}, \\
q^{(1)} & =\Theta(y, k, \gamma) e^{i k x+\gamma t}, \\
\Psi^{(1)} & =\Omega(y, z, k, \gamma) e^{i k x+\gamma t} .
\end{aligned}
$$

We substitute Eqs. (3.21)-(3.23) into Eqs. (3.18)-(3.20) and non-dimensionalize the system by dividing lengths by $d$, times by $\epsilon_{0} d / \sigma_{s}$ and charge densities by $\epsilon_{0} V / d$. We then write $D=\partial / \partial y$ and define new dimensionless quantities $\kappa=k d$ and $Q(y)=d^{2} D q^{(0)}(y) / \epsilon_{0} V$. The resulting dimensionless equations are

$$
\left(D^{2}-\kappa^{2}\right)\left(D^{2}-\kappa^{2}-\frac{\gamma}{\mathcal{P}}\right) \Lambda+\kappa^{2} \mathcal{R}\left(\Theta-Q \Omega_{s}\right)=0
$$

and

$$
\left(D^{2}-\kappa^{2}\right) \Omega_{s}-\gamma \Theta-Q \Lambda=0,
$$

where $\Omega_{s}=\left.\Omega\right|_{z=0}$. The three-dimensional Laplace equation, Eq. (3.18), becomes

$$
\nabla^{2}\left(\Omega e^{i \kappa x}\right)=\left[\frac{\partial^{2}}{\partial y^{2}}+\frac{\partial^{2}}{\partial z^{2}}-\kappa^{2}\right] \Omega=0,
$$

with Eq. (2.5) imposing the condition that

$$
\Theta=-\left.2 \frac{\partial \Omega}{\partial z}\right|_{z=0^{+}} .
$$

Eq. (3.26) is a two-dimensional Helmholtz equation in the half plane $x=0, z \geq 0$, which is perpendicular to the plane of the film. Eqs. (3.26) and (3.27) determine the rather complicated nonlocal coupling between the in-plane potential function $\Omega_{s}(y, \kappa, \gamma)=\Omega(y, 0, \kappa, \gamma)$ and the charge density function $\Theta(y, \kappa, \gamma)$.

Two dimensionless groups appear: $\mathcal{R}$, which plays the part of the Rayleigh number, and $\mathcal{P}$, which plays the part of the Prandtl number. In terms of the bulk, rather than surface, material parameters, they are given by 


$$
\mathcal{R}=\frac{\epsilon_{0}^{2} V^{2}}{\sigma \eta s^{2}}
$$

and

$$
\mathcal{P}=\frac{\epsilon_{0} \eta}{\rho \sigma d s}
$$

$\mathcal{R}$, the control parameter, is proportional to $V^{2}$. It is interesting to note that $\mathcal{R}$ is independent of $d$, the width of the film. The Prandtl-like parameter $\mathcal{P}$ may be regarded as the ratio $\tau_{q} / \tau_{v}$ of the two time scales in the problem, the charge relaxation time for thin films [8] $\tau_{q}=\epsilon_{0} d / \sigma s$ and the viscous relaxation time $\tau_{v}=\rho d^{2} / \eta$.

The effect of the nonlinear $y$ dependence of the derivative of the base state charge density $D q^{(0)}(y)$ is contained in the non-constant coefficient $Q(y)$. For the plate and wire electrode configurations, we find from Eqs. (2.7) and (2.14) that $Q(y)$ is given by

$$
Q_{p}(y)=\frac{8}{\pi\left(1-4 y^{2}\right)}
$$

and

$$
Q_{w}(y)=\frac{4}{\left(1-4 y^{2}\right)^{3 / 2}}
$$

respectively.

\section{Analogy to the Bénard Problem}

The above equations bear a strong analogy to the corresponding equations in the Bénard problem. The correspondence becomes complete if the nonlocal coupling of the charges and potentials given by Eq. (3.26) and (3.27), is suppressed by simply putting $q \propto \Psi_{s}$. Applying this assumption to the base state removes the nonlinear $y$ dependence of the charge density so that $Q(y) \equiv 1$. In fact, detailed analysis shows that $q$ is always nearly proportional to $\Psi_{s}$ in the central part of the film. This can be seen, for example, in Fig. 3 near $y=0$. If this proportionality is assumed to hold everywhere, then our continuity equation for charge, Eq. (3.4) becomes identical to the thermal diffusion equation in the Bénard problem. Under the 
same assumption, the force term $q \overrightarrow{\mathbf{E}}_{s}$ in Eq. (3.1) becomes proportional to $q \hat{\mathbf{y}}$, which is the form of the analgous term in the Bénard problem. Turning the argument around, one can say that the reason that our system does not reduce to the Bénard problem is because the charges and fields are nonlocally coupled via the the charge distribution's own self-field.

\section{The Compatibility Condition}

To find the conditions which $\mathcal{R}, \mathcal{P}, \kappa$ and $\gamma$ must satisfy for solutions to exist, we solve the linearized equations by means of various expansions. A crucial step that must be done numerically is the solution of the Helmholtz equation in the plane perpendicular to the film, Eq. (3.26), which necessarily involves a numerical relaxation calculation.

At the edges of the film, $y= \pm 1 / 2$, the rigid boundary conditions on the flow velocity $\overrightarrow{\mathbf{u}}^{(1)}$, given by Eq. (3.3), require that $\Lambda(y)$ satisfy the four conditions

$$
\Lambda( \pm 1 / 2)=D \Lambda( \pm 1 / 2)=0 .
$$

To ensure this, we expand $\Lambda(y)$ as

$$
\Lambda(y)=\sum_{m=1}^{\infty} A_{m} C_{m}(y)
$$

where the $C_{m}(y)$ are even Chandrasekhar functions [4],

$$
C_{m}(y)=\frac{\cosh \left(\lambda_{m} y\right)}{\cosh \left(\lambda_{m} / 2\right)}-\frac{\cos \left(\lambda_{m} y\right)}{\cos \left(\lambda_{m} / 2\right)} .
$$

Here $\lambda_{m}$ is the $m$ th root of $\tanh \left(\lambda_{m} / 2\right)+\tan \left(\lambda_{m} / 2\right)=0$ [15]. We can restrict the expansion to even functions because of the symmetry of the equations about $y=0$. Note that an expansion in $C_{m}$ has been shown to give a good description of the velocity field measured in experiments on electroconvection in smectic films [8]. Only relative amplitudes matter in Eq. (3.33), so we set $A_{1}=1$. It follows from linearity that we can also write $\Omega=\sum_{m} A_{m} \Omega_{m}$ and $\Theta=\sum_{m} A_{m} \Theta_{m}$, where $\Omega_{m}$ and $\Theta_{m}$ are the solutions corresponding to $\Lambda=C_{m}$. As above, we denote $\Omega_{s m}=\left.\Omega_{m}\right|_{z=0}$. 


\section{The potential function $\Omega_{\text {sm }}$ for $\gamma=0$}

Setting $\gamma=0$ and substituting $C_{m}$ for $\Lambda$ in Eq. (3.25) gives

$$
\left(D^{2}-\kappa^{2}\right) \Omega_{s m}=Q C_{m}
$$

which may be solved directly by Fourier expansion. Since $Q C_{m}$ is even, we expand

$$
Q C_{m}=\sum_{n=0}^{\infty} b_{m n} \cos (2 n \pi y)
$$

in which

$$
b_{m 0}=2 \int_{0}^{\frac{1}{2}} Q(y) C_{m}(y) d y
$$

and

$$
b_{m n}=4 \int_{0}^{\frac{1}{2}} Q(y) C_{m}(y) \cos (2 n \pi y) d y
$$

Using a similar Fourier expansion of $\Omega_{s m}$ and imposing the zero boundary conditions at $y= \pm 1 / 2$, we find

$$
\Omega_{s m}=\sum_{n=0}^{\infty} \frac{b_{m n}}{\left[(2 n \pi)^{2}+\kappa^{2}\right]}\left[\frac{(-1)^{n} \cosh (\kappa y)}{\cosh (\kappa / 2)}-\cos (2 n \pi y)\right] .
$$

To calculate $\Omega_{s m}$, we used a Romberg numerical integration scheme [16] to tabulate the integrals for $b_{m n}$ in Eqs. (3.37) and (3.38) for the each of the two electrode geometries, using $Q_{p}$ and $Q_{w}$ as given by Eqs. (3.30) and (3.31). We used an upper cutoff of $n=29$, which was dictated by the double precision accuracy of the Romberg scheme.

\section{The potential function $\Omega_{\text {sm }}$ for $\gamma \neq 0$}

For nonzero $\gamma$, we solved

$$
\left(D^{2}-\kappa^{2}\right) \Omega_{s m}=Q C_{m}+\gamma \Theta_{m}
$$

by an iterative scheme. We used the $\gamma=0$ solution, Eq. (3.39), to find a first approximation $\Omega_{s m}{ }^{[0]}$. From this, we calculated the corresponding approximate the charge density function 
$\Theta_{m}{ }^{[0]}$ using the relaxation algorithm described in Section IIID 3 below. Then $Q C_{m}+\gamma \Theta_{m}{ }^{[0]}$ was Fourier expanded in the same manner as $Q C_{m}$ in Eqs. (3.36)-(3.38) above. This expansion was used to find a series solution analogous to Eq. (3.39) for the next approximation $\Omega_{s m}{ }^{[1]}$, which was then relaxed to find $\Theta_{m}^{[1]}$. This sequence of steps was iterated until it converged for both $\Omega_{s m}$ and $\Theta_{m}$. The convergence criterion was a sum of the squares of 100 differences in successive iterates distributed on $0 \leq y \leq 1 / 2$. For $|\gamma| \leq 0.1$, the sum converged after 7 or 8 iterations to a precision limited by the Romberg integration scheme used to find the Fourier coefficients.

\section{The charge density function $\Theta_{m}$}

We solved the Helmholtz equation, Eq. (3.26), for $\Omega_{m}$ for each of the two electrode geometries, using a simple SOR algorithm [16]. In each case, the Dirichlet conditions on $\Omega_{m}$ for $-1 / 2 \leq y \leq 1 / 2$ and $z=0$ are given by $\Omega_{s m}$ (Eq. (3.39)) in the case of $\gamma=0$, or by the corresponding expression during iteration for $\gamma \neq 0$. Beyond the film, for $|y|>1 / 2$, $z=0$, we applied the Dirichlet condition $\Omega_{m}=0$ in the plate electrode case, and Neumann conditions $\left.\left(\partial \Omega_{m} / \partial z\right)\right|_{z=0}=0$ in the wire case.

Because $\Omega_{m}$ is even in $y$, it need only be relaxed in the first quadrant of the $y z$ plane. We used an $N \times N$ square lattice of cells in this quadrant, with $N_{f i l m}<N$ points between $y=0$ and $y=1 / 2$. On the outer edges of the lattice, we set $\Omega_{m}=0$ to enforce the zero boundary condition at infinity. Starting with $N=100$ and $N_{f i l m}=50$, we systematically increased $N$ and $N_{f i l m}$ is such a way that $N_{\text {film }} / N \rightarrow 0$. All the quantities calculated below showed a small residual monotonic variation with $N_{\text {film }}$; we removed this by plotting each against $1 / N_{\text {film }}$ and extrapolating to $1 / N_{\text {film }} \rightarrow 0$.

From the resulting $\Omega_{m}$, the charge density perturbation $\Theta_{m}$ was determined from Eq. (3.27) by taking the one-sided $z$ derivative numerically. $\Theta_{m}$ was therefore only known at $N_{\text {film }}$ lattice points across the film. For the purposes of integration, we used a Chebyshev interpolation [16] of these points. 


\section{The compatibility condition}

To find the general compatibility conditions on solutions, we substitute the expansions for $\Lambda, \Omega_{s}$ and $\Theta$ into Eq. (3.24) to get

$$
\sum_{m=1}^{\infty}\left[\left(D^{2}-\kappa^{2}\right)\left(D^{2}-\kappa^{2}-\frac{\gamma}{\mathcal{P}}\right) C_{m}+\kappa^{2} \mathcal{R}\left(\Theta_{m}-Q \Omega_{s m}\right)\right] A_{m}=0 .
$$

Multiplying by the Chandrasekhar function $C_{l}(y)$ and integrating from $y=-1 / 2$ to $y=+1 / 2$, we form inner products, denoted by $\langle\cdots\rangle$. Then Eq. (3.41) becomes a linear homogenous system with the determinant compatibility condition

$$
\left\|\left\langle C_{l}\left(D^{2}-\kappa^{2}\right)\left(D^{2}-\kappa^{2}-\frac{\gamma}{\mathcal{P}}\right) C_{m}\right\rangle+\kappa^{2} \mathcal{R}\left\langle C_{l}\left(\Theta_{m}-Q \Omega_{s m}\right)\right\rangle\right\|=0 .
$$

After some simplification, this can be written as

$$
\left\|\left(\lambda_{m}^{4}+\kappa^{4}\right) \delta_{l m}-2 \kappa^{2} X_{l m}+\kappa^{2} \mathcal{R} F_{l m}-\frac{\gamma}{\mathcal{P}}\left(X_{l m}-\kappa^{2} \delta_{l m}\right)\right\|=0,
$$

where $F_{l m}=\left\langle C_{l}\left(\Theta_{m}-Q \Omega_{s m}\right)\right\rangle$. The matrix elements $X_{l m}$ are given analytically by $\llbracket$

$$
\begin{aligned}
X_{l m} & =\left\langle C_{l}^{\prime \prime} C_{m}\right\rangle \\
& =\left.\frac{2}{\lambda_{l}^{4}-\lambda_{m}^{4}}\left(C_{l}^{\prime \prime \prime} C_{m}^{\prime \prime}-C_{m}^{\prime \prime \prime \prime} C_{l}^{\prime \prime}\right)\right|_{y=\frac{1}{2}} \quad \text { when } l \neq m \\
& =\left.\frac{1}{\lambda_{m}^{4}}\left(\frac{1}{2} C_{m}^{\prime \prime \prime} C_{m}^{\prime \prime}-\frac{1}{4}\left(C_{m}^{\prime \prime \prime}\right)^{2}\right)\right|_{y=\frac{1}{2}} \quad \text { when } l=m,
\end{aligned}
$$

where $C_{m}^{\prime \prime}=D^{2} C_{m}(y)$, etc. The matrix elements $F_{l m}(\kappa, \gamma)$ were evaluated numerically for

each electrode configuration using Romberg integration [16]. The divergences in $Q(y)$ at the edges of the film are overcome because $C_{l}(y)$ goes to zero sufficiently fast at $y= \pm 1 / 2$. The functions $\Theta_{m}$ and $\Omega_{s m}$ are simple smooth functions for the first few values of $m$ and are straightforward to integrate numerically.

\section{E. Marginal Stability}

To find the conditions for marginal stability, we set the growth rate of the perturbations $\gamma$ equal to zero in the compatibility condition, Eq. (3.43). The Prandtl-like dimensionless 
group $\mathcal{P}$ drops out, so that the marginal stability conditions are independent of $\mathcal{P}$, just as is the case in the Bénard problem. Eq. (3.43) then implicitly defines the marginal stability curve $\mathcal{R}=\mathcal{R}_{o}(\kappa)$. We proceeded as follows. Choosing a value of $\kappa$, we set $l=m=1$ and calculate $F_{11}(\kappa)$. Then Eq. (3.43) can be simply solved to get the first approximation $\mathcal{R}_{o}^{[1]}(\kappa)$. We then find $F_{l m}(\kappa)$ for $l, m=1,2$ and search near $\mathcal{R}_{o}^{[1]}(\kappa)$ for roots of the $2 \times 2$ determinant, Eq. (3.43), to find $\mathcal{R}_{o}^{[2]}(\kappa)$. We can then use $A_{1}=1$ to find $A_{2}$ in Eq. (3.33) by backsubstitution. We carried this algorithm to the third order in the Chandrasekhar expansion, for which the maximum value of $\left|A_{3}\right|$ is of order $10^{-2}$ and the resulting neutral curve $\mathcal{R}_{o}(\kappa)$ no longer changes significantly. Fig. 14 shows the amplitudes $A_{2}$ and $A_{3}$ for the wire case, relative to $A_{1}=1$. It is clear that the higher terms in the Chandrasekhar expansion contribute very little to the sum in Eq. (3.33).

Fig. 5 shows the neutral curve for the plate and wire electrode configurations. The minima of these curves define the critical values $\kappa_{c}$ and $\mathcal{R}_{c}=\mathcal{R}_{o}\left(\kappa_{c}\right)$ for each case. These values are listed in Table $\mathbf{\text { I. }}$. We find that both neutral curves give $\kappa_{c}$ between 4 and 5 , but that $\mathcal{R}_{c}$ is lower for the wire electrode case. This is apparently due to the steeper slope of $q^{(0)}(y)$, evident in Fig. 3, for the case of wire electrodes. Neither value of $\kappa_{c}$ is particularly close to the Bénard value of 3.117, but they are in reasonable agreement with the value determined from the smectic film experiments [6,8,10], as discussed in section IV below.

We can define a length scale $\xi_{0}$ in terms of the curvature of $\mathcal{R}_{o}(\kappa)$ near $\kappa_{c}$ [1, 2, 20]:

$$
\xi_{0}^{2}=\left.\frac{1}{2} \frac{d^{2} \epsilon_{c}}{d \kappa^{2}}\right|_{\kappa=\kappa_{c}},
$$

where $\epsilon_{c}=\left(\mathcal{R}_{o}(\kappa) / \mathcal{R}_{c}\right)-1$. This length will appear as a coefficient in an amplitude equation description of the convection pattern near onset [1,2,5]. To find $\xi_{0}$ accurately, we fit $\epsilon_{c}$ to a parabola over a range $\kappa=\kappa_{c} \pm \Delta \kappa$ and then systematically reduced $\Delta \kappa$ until the value of $\xi_{0}$ taken from the fit became independent of $\Delta \kappa$. This corresponded to a fitting range $\epsilon_{c} \leq 5 \times 10^{-4}$. The values of $\xi_{0}$, given in Table [1, were slightly dependent on the electrode configuration. 


\section{F. The Linear Growth Rate $\gamma$}

Returning to the full compatibility condition Eq. (3.43) with $\gamma \neq 0$, we consider the behavior of the growth rate $\gamma$ of the linear modes near the critical values of $\mathcal{R}$ and $\kappa$. The time scale $\tau_{0}$, defined by

$$
\tau_{0}^{-1}=\left.\frac{\partial \gamma(\epsilon)}{\partial \epsilon}\right|_{\kappa=\kappa_{c}, \epsilon=0}
$$

where $\epsilon=\left(\mathcal{R} / \mathcal{R}_{c}\right)-1$, will also appear in an amplitude equation description of the pattern near onset [1,:2,5].

The matrix element $F_{l m}(\kappa, \gamma)$ is rather expensive to calculate for $\gamma \neq 0$, because we must use the iteration scheme outlined in Section [IID 2. It is most computationally efficient to choose a value of $\gamma$, fix $\kappa=\kappa_{c}$, and then solve Eq. (3.43) for $\mathcal{R}$. This was done for ten values of $\gamma$ in the range $-0.1 \leq \gamma \leq+0.1$, using three Chandrasekhar modes. The results depend on $\mathcal{P}$. The resulting function $\gamma(\epsilon)$ is very nearly linear in $\epsilon$ with a $\mathcal{P}$-dependent slope and $\gamma(0)=0$. We determined $\tau_{0}$ from polynomial fits to $\gamma(\epsilon)$ for $\mathcal{P} \geq 0.01$. The results are only slightly dependent on electrode configuration. $\tau_{0}$ is plotted as a function of $\mathcal{P}$ for wire electrodes in Fig. 6. For $\mathcal{P}>1, \tau_{0}$ tends towards the limiting values given in Table [.

\section{DISCUSSION}

The stability analysis presented above demonstrates that a thin, weakly conducting suspended fluid film becomes unstable to spatially periodic convective flow if a sufficiently large voltage is applied across the film. Since our analysis is linear, it cannot describe the convection pattern above onset, but it does provide important information about the onset of convection. In this Section, we discuss the theoretical results in the light of previous experiments on smectic [6, [7,8,10] and nematic [11] films.

Eq. (3.28), combined with the neutral curve, predicts that the onset of convection occurs at a critical voltage proportional to the film thickness $s$, and independent of the film width $d$, given by 


$$
V_{c}=\frac{s}{\epsilon_{0}} \sqrt{\sigma \eta \mathcal{R}_{c}}
$$

The dependence of $V_{c}$ on $\sqrt{\sigma \eta}$ follows inevitably from dimensional analysis. Faetti et al. also found a critical voltage proportional to $s \sqrt{\sigma \eta}$ and independent of $d$ from a highly simplified model of the "vortex mode" observed by them in nematic films [11].

In experiments on convection in smectic films, $V_{c}$ has been found to be proportional to $s$ for films up to about 20 molecular layers (i.e., about $63 \mathrm{~nm}$ ) thick [10]. For larger $s, V_{c}$ grows somewhat more slowly. This may be a sign that layer-over-layer shears in the $z$ direction exist for thicker films; such flows are not accounted for in our calculation. A linear dependence of $V_{c}$ on $s$ has also been observed in experiments on nematic films [11]. The nematic films are much thicker than the smectic films, and have significant thickness nonuniformities. The also exhibit slow flows even below the onset of convection, making $V_{c}(s)$ rather difficult to measure.

Our most recent experiments on smectic films [10] show no dependence of $V_{c}$ on $d$ for films with $d$ between 0.7 and $2.0 \mathrm{~mm}$, with thicknesses $s$ between two and 25 molecular layers, that is, between $6.3 \mathrm{~nm}$ and $80 \mathrm{~nm}$. This is consistent with the prediction of Eq. (4.1). Over about the same range of thickness, as noted above, $V_{c}$ is also proportional to $s$, as predicted. A weak variation of $V_{c}$ with $d$ was, however, observed in our earlier work [8] for $d$ in the larger range of $0.36 \mathrm{~mm}$ to $3.5 \mathrm{~mm}$. This work used a thicker film (107 molecular layers, or $340 \mathrm{~nm}$ ) and a slightly different electrode configuration, with guard electrodes outside the main electrode wires. These features may have contributed some $d$-dependent three-dimensional effects.

The wavenumber at onset observed in smectic film experiments is [10 $k_{c}^{\text {expt }}=4.94 \pm$ $0.03 d^{-1}$. The measured value of $d$ is uncertain to $\pm 5 \%$, so this result yields a measured dimensionless wavenumber $\kappa_{c}^{\text {expt }}=4.94 \pm 0.25$. This is in satisfactory agreement with the value of $\kappa_{c}=4.74$ found from the minimum in the calculated neutral curve for wire electrodes. At present, no data is available for comparison to the predictions for the plate electrode geometry. 
It is interesting to note that the charge relaxation time $\tau_{q}=\epsilon_{0} d / \sigma s$ appropriate to thin conducting films emerges as the natural unit of time in our analysis. As discussed in Ref. [8], in a thin film the relaxation time is greater than the bulk value $\epsilon / \sigma$ by the factor $d / \epsilon_{r} s$, where $\epsilon_{r}$ is the relative permittivity of the fluid. This is a consequence of the restricted geometry, and the fact that the fields lie in the free space outside the film. The wave number of the convection pattern observed at onset changes when the film is driven with ac voltages for frequencies much larger than $1 / \tau_{q}$. It would be interesting to modify our analysis to allow for time-periodic driving voltages.

It is often useful to describe patterns near onset with an equation for the slowly varying amplitude $A$ of the pattern. For one-dimensional systems which are symmetric under $A \rightarrow$ $-A$, the appropriate amplitude equation is the Ginzburg-Landau equation [1,2],

$$
\tau_{0} \frac{\partial A}{\partial t}=\epsilon A-g|A|^{2} A+\xi_{0}^{2} \frac{\partial^{2} A}{\partial x^{2}}
$$

Here the amplitude $A$ can be taken as the amplitude of the convective velocity field, and $g$ governs the nonlinear saturation of the amplitude. $\xi_{0}$ and $\tau_{0}$ are characteristic length and time scales introduced earlier. We have previously demonstrated that measurements near the onset of convection in smectic films can be described well by Eq. (4.2) with $\epsilon=\left(V / V_{c}\right)^{2}-1[8,10]$. The onset of convection occurs at a supercritical bifurcation, and the $\epsilon$ dependence of the flow velocity above onset, the behavior of the amplitude of convection near a lateral boundary and the relaxation of the pattern amplitude after sudden changes in $\epsilon$ are all well described by fits to Eq. (4.2). Eq. (4.2) can also be derived from the full electrohydrodynamic equations of motion presented above [13.

Our analysis gives theoretical results for $\xi_{0}$ and $\tau_{0}$, as discussed above. Our predicted value for correlation length is $\xi_{0}=0.285$, which is about $20 \%$ smaller than the experimental value $\llbracket 10$ of $\xi_{0}^{\text {expt }}=0.36 \pm 0.03$. This is in fair, but not completely satisfactory, agreement. To arrive at both $\kappa_{c}^{\text {expt }}$ and $\xi_{0}^{\text {expt }}$, the experimental measurements were made nondimensional by dividing by the measured film width $d$, which is known to within about $5 \%$. The main obstacle to making quantitative comparisons to our predictions for $V_{c}(s)$ and $\tau_{0}$ are the 
poorly known material parameters $\sigma$ and (especially) $\eta$. These appear in the expression for the slope $V_{c}(s) / s$, and are also required for calculating nondimensional times. Realistic smectic films are sufficiently viscous that they have values of $\mathcal{P} \gg 1$, so that we expect the infinite- $\mathcal{P}$ limit of $\tau_{0}$ to apply. The conductivity $\sigma$ for the doped smectic liquid crystal used in our experiments has been measured at $1 \mathrm{kHz}$ in a bulk sample [17] and at dc in an annular film [18]. Over this frequency range, it changes by a factor of three. To get agreement between $\tau_{0}^{\text {expt }}$ and our theoretical value requires a value of $\sigma$ which lies between the dc and $1 \mathrm{kHz}$ measurements. Agreement with the $V_{c}(s) / s$ data [10, however, requires using a value of $\eta$ a factor of 20 larger than that estimated by extrapolating measurements of $\eta$ made in the higher temperature nematic phase [19]. This discrepancy may be a result of neglecting the effects of air drag on the moving film, which are likely to be important for thin, fast-moving films [20].

The instability we have described occurs in thin films of fluids which are isotropic in the plane of the film. It should also exist for anisotropic fluid films near dc, for example in films of smectic $\mathrm{C}$ and $\mathrm{C}^{*}$ materials. In smectic $\mathrm{C}$ materials, the molecules are tilted with respect to the layer planes, so the layers are two-dimensional analogs of a nematic fluid. Smectic $\mathrm{C}^{*}$ materials have an additional broken symmetry which allows a spontaneous electric dipole moment in the plane of the layers. Flows in these materials will involve strong orientational effects. It should be straightforward to generalize our analysis to the anisotropic case, which may lead to interesting new effects. Recently, it has been suggested that electroconvection, driven by the analog of the Carr-Helfrich mechanism which operates in negative dielectric anisotropy nematics [3], may occur in smectic $\mathrm{C}$ films under ac voltages [21]. If materials with the right parameters exist, it seems likely that the new instabilities will coexist or compete at low frequencies with the instability we have considered here. Something of this sort is observed in nematic films [11,22] in which both a "vortex" and a "domain" mode are found. 


\section{CONCLUSION}

We have presented a linear stability analysis for the onset of electroconvection in a thin conducting fluid with two free surfaces. We found the neutral stability curve $\mathcal{R}_{o}(\kappa)$, along with its critical values $\mathcal{R}_{c}$ and $\kappa_{c}$, and the correlation length $\xi_{0}$ implied by its curvature near $\kappa_{c}$. The linear growth rate was used to find the characteristic time $\tau_{0}$. This was done for two simple electrode configurations. These results were compared with experiments, mainly on smectic A films. Several generalizations of this analysis were suggested.

\section{ACKNOWLEDGMENTS}

We would like to thank S. S. Mao and T. Molteno for interesting discussions, and J.T. Gleeson for making conductivity measurements. This research was funded by the Natural Sciences and Engineering Research Council of Canada. 


\section{REFERENCES}

[1] M.C. Cross and P.C. Hohenberg, Rev. Mod. Phys. 65, 851 (1993).

[2] G. Ahlers, in Lectures in the Sciences of Complexity, edited by D. Stein (Addison-Wesley, Reading, 1989), p. 175.

[3] L. Kramer and W. Pesch, in Pattern Formation in Liquid Crystals, A. Buka and L. Kramer eds. (Springer, New York, 1995), and Annual Rev. Fluid Mech., 27, 515 (1995).

[4] S. Chandrasekhar, Hydrodynamic and Hydromagnetic Stability, (Oxford University Press, London, 1961).

[5] M. A. Dominguez-Lerma, G. Ahlers and D. S. Cannell, Phys. Fluids, 27, 856 (1984).

[6] S. W. Morris, J.R. de Bruyn, and A.D. May, Phys. Rev. Lett., 65, 2378 (1990).

[7] S. W. Morris, J. R. de Bruyn, and A. D. May, J. Stat. Phys., 64, 1025 (1991).

[8] S. W. Morris, J.R. de Bruyn, and A.D. May, Phys. Rev. A, 44, 8146 (1991).

[9] S. S. Mao, J. R. de Bruyn, Z. A. Daya and S. W. Morris, Phys. Rev. E, to be published.

[10] S. S. Mao, J. R. de Bruyn and S. W. Morris, Physica A, to be published.

[11] S. Faetti, L. Fronzoni, and P. Rolla, J. Chem. Phys., 79, 5054 (1983).

[12] W. V. R. Malkus and G. Veronis, Phys. Fluids, 4, 13 (1961).

[13] V. B. Deyirmenjian, Z. A. Daya and S. W. Morris, unpublished.

[14] I. Sneddon, Mixed Boundary Value Problems in Potential Theory (North-Holland, Amsterdam, 1966), p 103.

[15] There is an error in Ref. [8]: the sign is incorrect in Eq. (B4) and $\alpha$ and $\beta$ are interchanged in Eqs. (B4) and (B5).

[16] W. H. Press, S. A. Teukolsky, W. T. Vetterling and B. P. Flannery, Numerical Recipes in 
C, (Cambridge University Press, Cambridge, 1992).

[17] J. T. Gleeson, private communication.

[18] Z. A. Daya, unpublished.

[19] H. Kneppe, F. Schneider, and N.K. Sharma, Ber. Bunsenges. Phys. Chem., 85, 784 (1981).

[20] Y. Couder, J. M. Chomaz, and M. Rabaud, Physica D, 37, 406 (1989).

[21] S. Ried, H. Pleiner, W. Zimmermann and H. Brand, Phys. Rev. E, 53, 6101 (1996).

[22] S. Faetti, L. Fronzoni, and P. Rolla, J. Chem. Phys., 79, 1427 (1983). 


\section{TABLES}

TABLE I. Numerical results.

\begin{tabular}{lcc}
\hline \hline electrode geometry & wires & plates \\
\hline critical wavenumber, $\kappa_{c}$ & 4.744 & 4.223 \\
critical control parameter, $\mathcal{R}_{c}$ & 76.77 & 91.84 \\
correlation length, $\xi_{0}$ & 0.2843 & 0.2975 \\
time scale, $\tau_{0}(\mathcal{P}=\infty)$ & 0.351 & 0.372 \\
\hline \hline
\end{tabular}




\section{FIGURES}
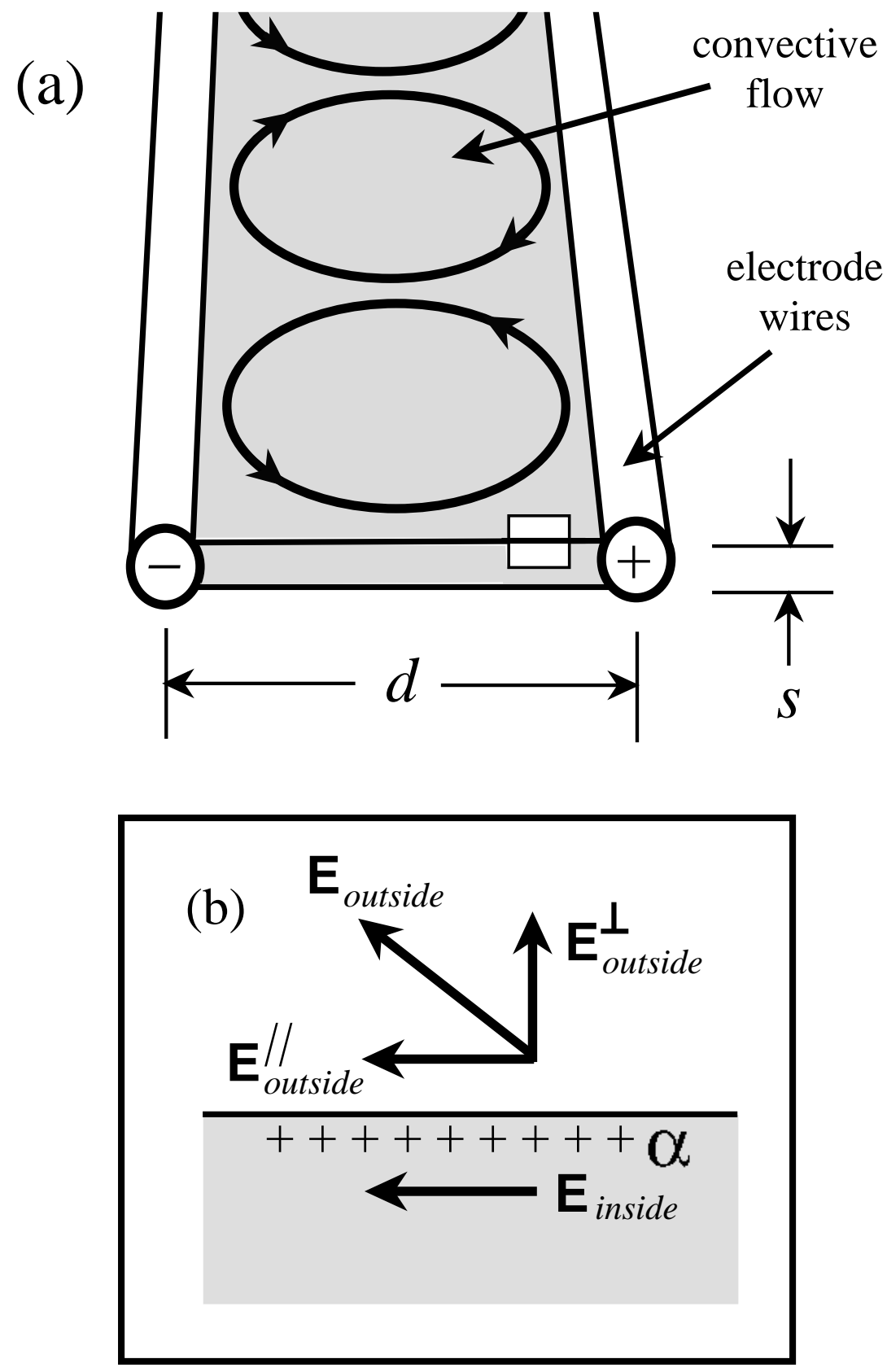

FIG. 1. (a) A schematic of the film and electrodes, as used in smectic experiments [8]. The film and electrode are shown enormously exaggerated in thickness; in fact $s / d \approx 10^{-5}$. (b) Schematic illustration of the fields inside and outside the film in the small box in part (a). $\alpha$ is a surface charge density. 

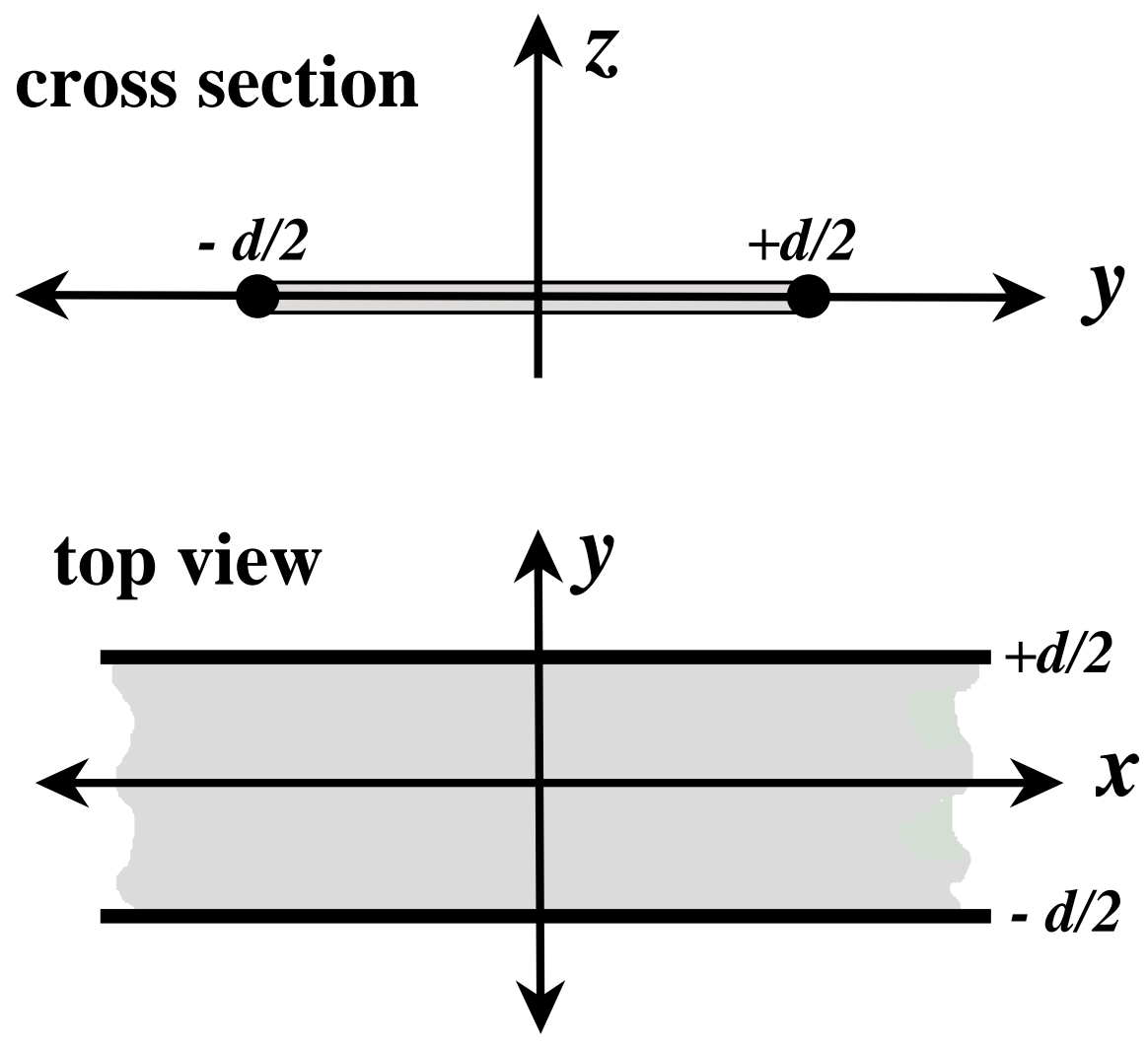

FIG. 2. The coordinates used in the analysis, in which the film is treated as a two-dimensional sheet. 


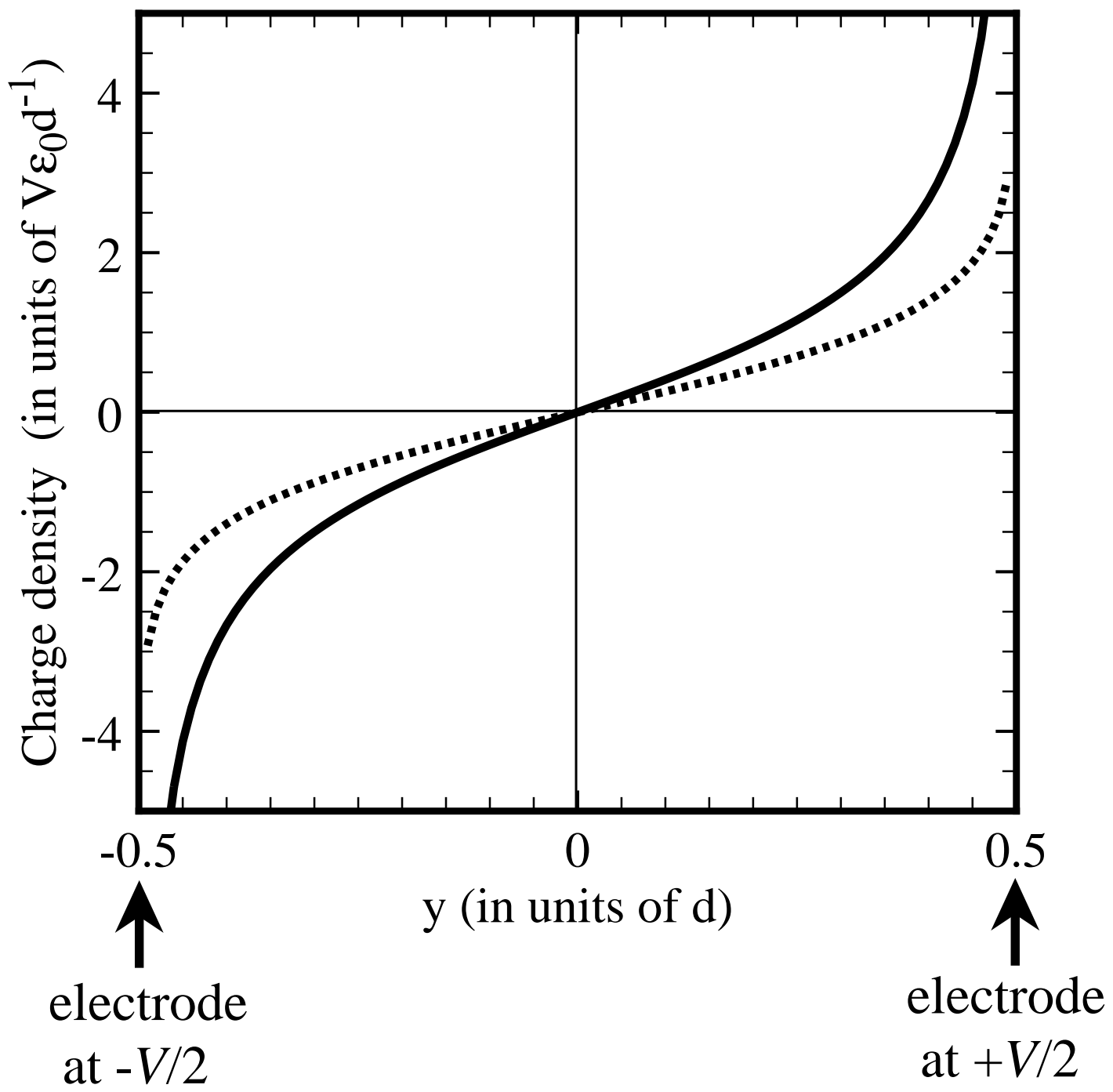

FIG. 3. The surface charge densities for plate (dashed line) and wire (solid line) electrodes. 


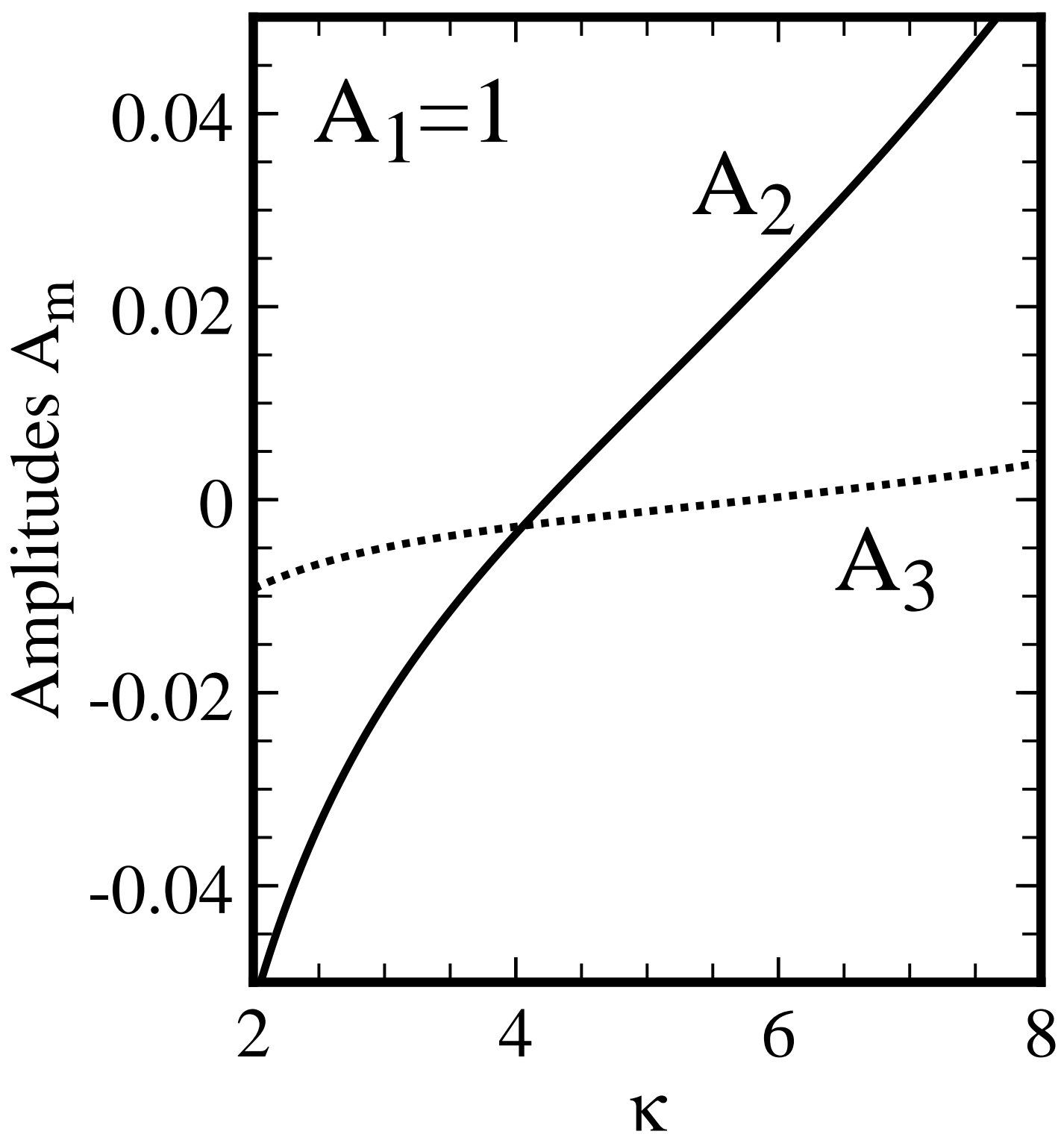

FIG. 4. The amplitudes $A_{2}$ and $A_{3}$ of the second and third terms of the expansion for $\Lambda(y)$, Eq. (3.33). 


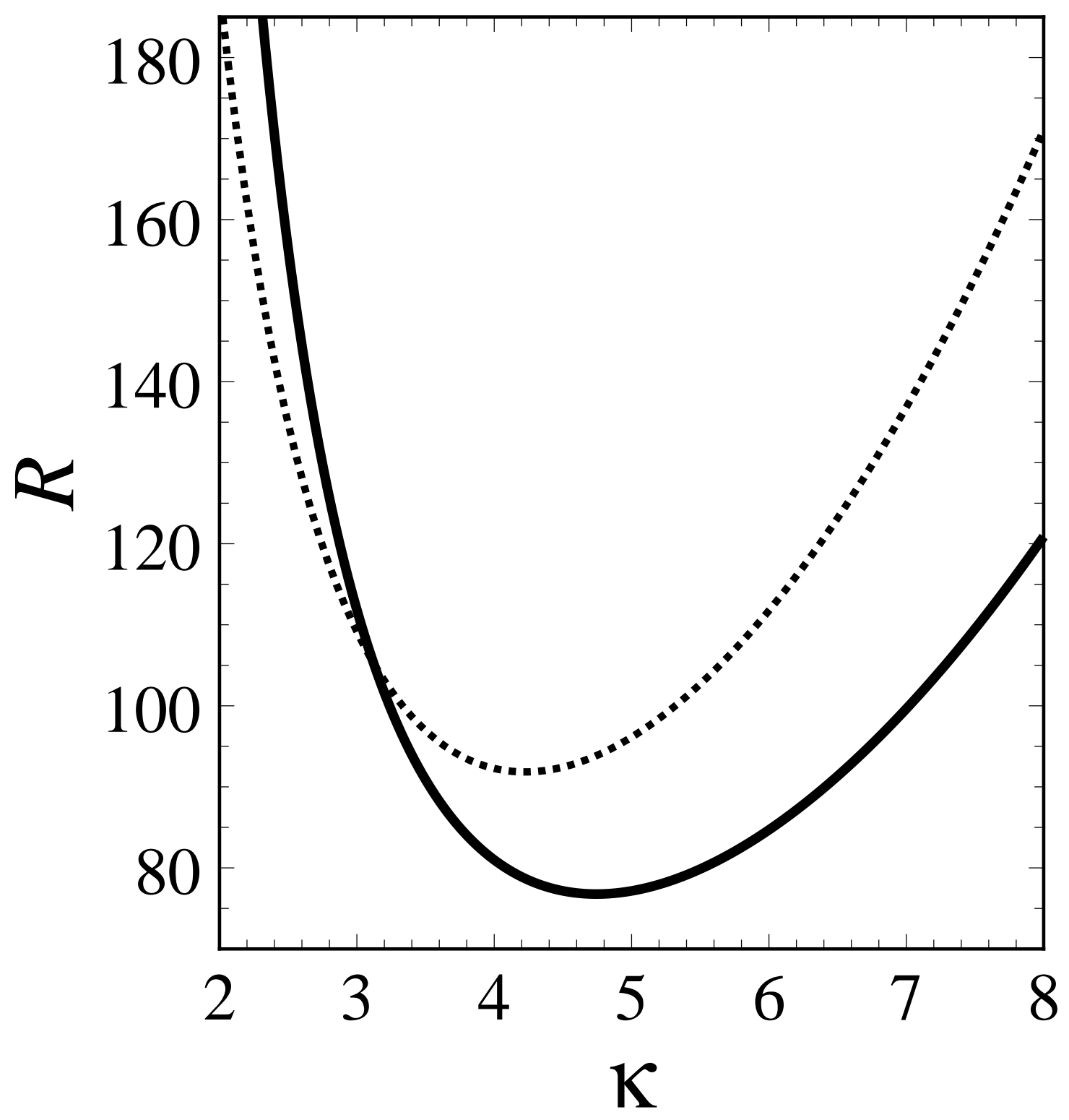

FIG. 5. The neutral curve for plate (dashed line) and wire (solid line) electrodes. 


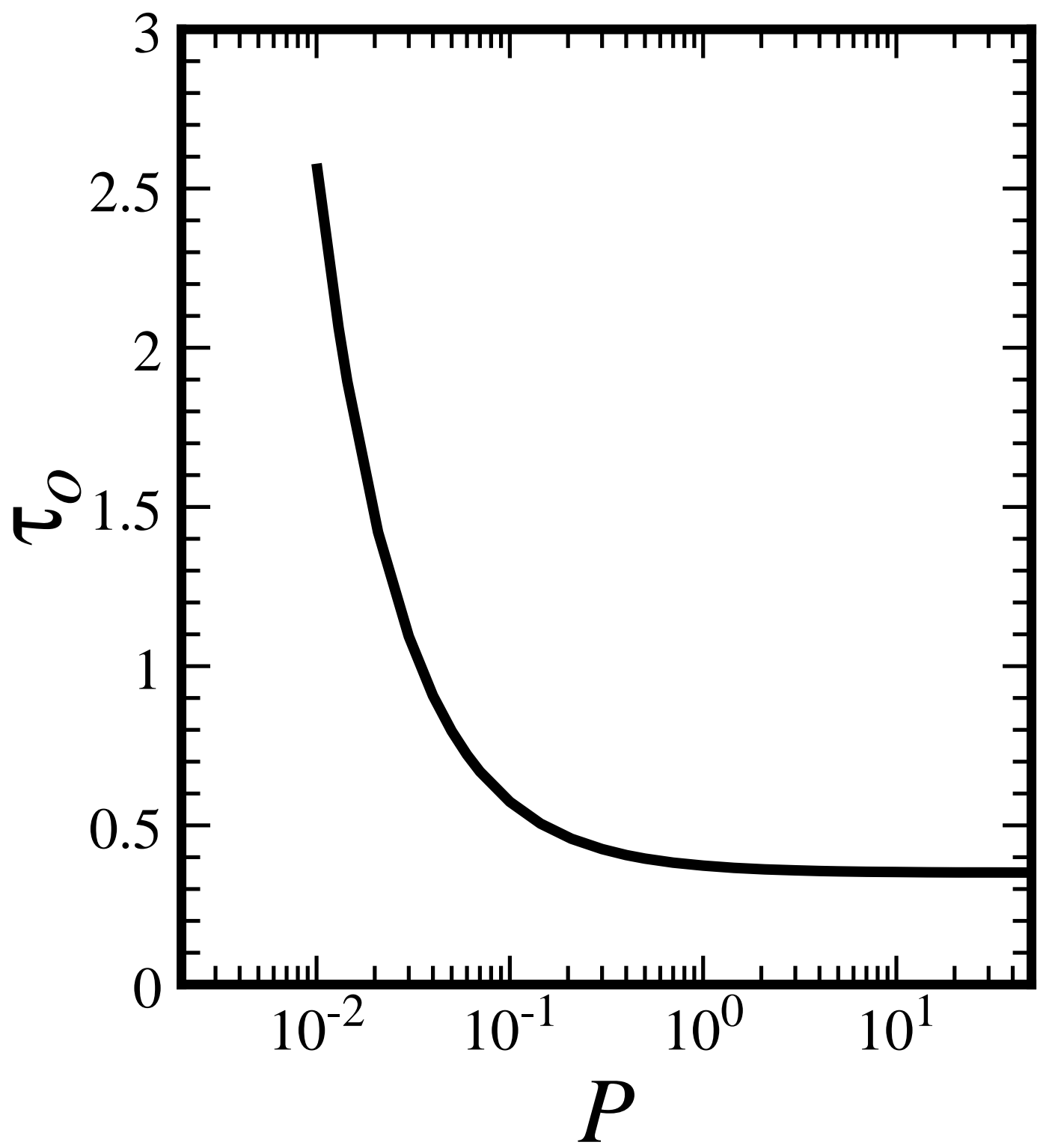

FIG. 6. $\tau_{0}$ as a function of $\mathcal{P}$ for wire electrodes. 